\title{
Erratum
}

\section{Boundary cohomology of Shimura varieties, II. Hodge theory at the boundary}

\author{
M. Harris, S. Zucker
}

Invent. math. 116, 243-308 (1994)

There is a potentially misleading error in the statement of Theorem (5.6.10). It should read:

(5.6.10) Theorem. There is a decomposition of mixed Hodge structures

$H_{d n}^{i}\left(Z_{\Sigma}(R), \tilde{\mathbf{V}}_{(P)}\right) \simeq \bigoplus_{j, k} \bigoplus_{w \in W^{1, P}, /(w)=j} H^{l-j-k}\left(M_{P},\left(\tilde{\mathbf{V}}_{w * \mu}\right)_{h}\right) \otimes_{\mathbb{C}} H^{k}\left(\Gamma_{/, R},\left(V_{w * \mu}\right)_{l}\right)$, where $H^{k}\left(\Gamma_{f, R},\left(V_{w * \mu}\right)_{\ell}\right)$ has the pure Hodge structure of type $(0,0)$.

The mistake is that in the article, "type $(k, k)$ " appears instead of "type $(0,0)$ ". The latter is correct because the simplicial and degree filtrations cancel (moreover, the Hodge filtration is trivial!). We wish to point out that the result is announced in [H:(3.5.2)], where it is stated correctly.

On the other hand, the error is copied into [Z:App. B], part of a preprint collection, and even propagates a little further. Recall that in (5.7), we briefly treated possible applications to the non-existence of ghost classes. The calculations in [Z] that assert that there are no ghost classes in the rank two symplectic case, when redone correctly, give incomplete results (one must assume generic or trivial coefficients). However, they do show that the presence of ghost classes in this case imply the existence of Tate structures in the cohomology of the Shimura variety.

[H] Harris, M.: Hodge-de Rham structures and periods of automorphic forms. In: Jannsen, et al. (eds.) Motives, PSPM 55(2) 573-624 (1994)

[Z] Zucker, S.: On the boundary cohomology of locally symmetric varieties. In: Algebraic Geometry Symposium (Saitama Univ.), 198-232 (1993) 